\title{
Regional Myocardial Blood Flow during
}

\section{Graded Treadmill Exercise in the Dog}

\author{
Robert M. Ball, Robert J. Bache, Frederick R. Cobb, and \\ Joseph C. Greenfield, JR. \\ From the Veterans Administration Hospital and the Department of Medicine, \\ Division of Cardiology, Duke University Medical Center School of Medicine, \\ Durham, North Carolina 27705
}

\begin{abstract}
A B S T R A C T Regional myocardial blood flow was measured in nine dogs at rest and during three levels of treadmill exercise by using left atrial injections of $7-10-\mu \mathrm{m}$ radioactive microspheres. At rest, heart rate was $76 \pm 3$ beats/min (mean \pm SEM), mean left ventricular myocardial flow was $0.94 \pm 0.09 \mathrm{ml} / \mathrm{min} / \mathrm{g}$ and endocardial flow (endo) exceeded epicardial flow (epi) in all regions (endo/epi $=1.12-1.33$ ). When treadmill exercise was regulated to increase heart rates from $152 \pm 3$ to $190 \pm 3$ to $240 \pm 6$ beats/min, myocardial blood flow (MBF) to all regions of the left ventricle increased linearly with heart rate (HR) from $1.83 \pm 0.11$ to $2.75 \pm$ 0.22 to $3.90 \pm 0.26 \mathrm{ml} / \mathrm{min} / \mathrm{g}$ (MBF $=0.0175 \mathrm{HR}-0.523$ $\pm 0.614, r=0.87$ ). Exercise abolished the gradient of blood flow favoring the left ventricular endocardium at rest, so that the endo/epi flow ratios were not significantly different from 1.00 . Right ventricular flows were consistently less than corresponding left ventricular flows, but showed a similar linear increase with heart rate. Right ventricular endo/epi ratios were not different from 1.00 either at rest or during exercise. Thus, exercise resulted in increased myocardial blood flow to all regions of the left and right ventricles with maintenance of subendocardial flow equal to subepicardial flow.
\end{abstract}

\section{INTRODUCTION}

Although several studies have shown that proximal coronary artery blood flow increases in response to ex-

Mr. Ball is a Senior Medical Student, Duke University School of Medicine, Durham, North Carolina 27710. Dr. Cobb is a Clinical Investigator, Veterans Administration Hospital, Durham, North Carolina 27705. Dr. Greenfield is recipient of Research Career Development Award 1-K3HL-28112 from the U. S. Public Health Service.

Received for publication 12 June 1974 and in revised form 29 August 1974.

The Journal of Clinical Investigation Volume 55 January 1975.43-49 ercise (1-4), regional myocardial blood flow has not been measured previously during exercise. Since perfusion of the subendocardial myocardium occurs principally during diastole, uniform transmural myocardial perfusion requires a gradient of coronary vascular resistance during diastole with resistance lower in the subendocardium than the subepicardium. Exercise results not only in tachycardia with shortening of the interval of diastole, but also in coronary vasodilation in response to increased myocardial oxygen consumption $(5,6)$. Because the generalized coronary vasodilation resulting from exercise might limit the capacity of the subendocardial vasculature to maintain resistance sufficiently lower than in the subepicardium to compensate for the shortened diastolic perfusion period, it is possible that subendocardial perfusion could be compromised during exercise. This concept is supported by previous studies in open-chest dogs which have suggested that the subendocardial vasculature may be maximally dilated at rest and thus unable to increase blood flow in response to increased myocardial oxygen demand $(7,8)$. Consequently, the present study was performed to measure regional myocardial blood flow during exercise to assess the transmural distribution of myocardial blood flow.

\section{METHODS}

Nine adult mongrel dogs weighing $22-34 \mathrm{~kg}$ were anesthetized with sodium thiamylal $(40 \mathrm{mg} / \mathrm{kg})$ and ventilated with a Harvard respirator. ${ }^{1}$ A left thoracotomy was performed in the fourth intercostal space. A polyvinyl chloride catheter, $3 \mathrm{~mm} O D$, was inserted into the left atrial cavity via the atrial appendage and secured with a purse-string suture. A polyvinyl chloride catheter, $3.5 \mathrm{~mm} \mathrm{OD,} \mathrm{was}$ inserted into the root of the aorta via the left internal thoracic artery. Both catheters were filled with heparin

\footnotetext{
${ }^{1}$ Harvard Apparatus Co., Inc., Millis, Mass.
} 
and tunneled to a subcutaneous pouch at the base of the neck. 7-10 days after surgery, training was begun to teach the dogs to run on a motor-driven treadmill.: Studies were performed 17-21 days later when dogs were free from fever, anemia, or other evidence of ill health.

On the day of the study, the left atrial and aortic catheters were exteriorized from the subcutaneous pouch using $2 \%$ lidocaine infiltration anesthesia. The arterial catheter was attached to a Statham P37Db pressure transducer mounted on the dog's side and a polyethylene tube connected to a constant rate withdrawal pump." The left atrial catheter was connected via a stiff polyethylene tube to a Statham $\mathrm{P} 23 \mathrm{Db}$ transducer mounted on the treadmill cage at the level of the left atrium. Phasic and mean aortic and mean left atrial pressures were recorded continuously on a directwriting oscillographic system. ${ }^{5}$

Measurements of regional myocardial blood flow were made by using serial injections of carbonized microspheres 7-10 $\mu \mathrm{m}$ in diameter labeled with gamma-emitting nuclides ${ }^{141} \mathrm{Ce},{ }^{51} \mathrm{Cr},{ }^{85} \mathrm{Sr}$, and ${ }^{16} \mathrm{Sc}$. The microspheres were diluted in $10 \%$ low molecular weight dextran so that $1.5 \mathrm{~cm}^{3}$, the volume injected, contained approximately three million microspheres. This dosage was selected so that during resting conditions each gram of myocardium would receive a minimum of 500 microspheres assuming a coronary blood flow of $5 \%$ of the cardiac output and heart weight of 150-200 g. This number of microspheres has been shown to minimize error due to random distribution (9). Serial injections of this quantity of microspheres resulted in no measurable change in heart rate or blood pressure in the dogs included in this study. Before each injection the microspheres were thoroughly mixed by alternate agitation for at least $15 \mathrm{~min}$ in an ultrasonic bath and Vortex agitator.? Complete dispersion was verified by microscopic examination of a drop of the suspension. During each intervention, $1.5 \mathrm{~cm}^{8}$ of the microsphere suspension was injected into the left atrium over a 15-s interval via the previously implanted catheter and flushed with $10-15 \mathrm{~cm}^{8}$ of warm isotonic saline. Beginning at the start of injection, a reference sample of arterial blood was withdrawn from the aortic catheter at a constant rate of $15.0 \mathrm{ml} / \mathrm{min}$ for $90 \mathrm{~s}$. Hematocrits (36$48 \%$ ) were measured before and after each study and did not vary appreciably during the study.

Measurements of myocardial blood flow were made during quiet resting conditions while standing, and during light, moderate, and heavy exercise on the treadmill. Light exercise was defined as the speed and grade necessary to increase heart rates to $140-170$ beats $/ \mathrm{min}$. Moderate exercise was regulated to increase heart rates to $180-210$ beats/ min. Heavy exercise was the level of exercise necessary to achieve heart rates greater than 220 beats/min. Mean speed and grade for each exercise level were: light exercise $2.7 \mathrm{mph}$ and $1 \%$ grade; moderate exercise $4.6 \mathrm{mph}$ and $5 \%$ grade; and heavy exercise $6.4 \mathrm{mph}$ and $11 \%$ grade.

Microspheres were injected $45 \mathrm{~s}$ after a hemodynamic steady state was achieved as monitored by heart rate and blood pressure. The 45-s time interval was chosen because previous investigators have shown that heart rate and coronary artery blood flow reach a steady state 10-20 s

Model 1849-D, Quinton Instruments, Seattle, Wash.

'Statham Instruments, Inc., Oxnard, Calif.

- Model 1210, Harvard Apparatus Co., Inc.

- Model 7700, Hewlett-Packard Co., Palo Alto, Calif.

- Nuclear Products Div., 3M Co., St. Paul, Minn.

${ }^{7}$ Scientific Industries, Inc., Mineola, N. Y. after the onset of exercise (1-4). Heart rate, mean arterial pressure, and mean left atrial pressure were measured directly from the recording during rest and each level of exercise.

At the completion of each study the dog was anesthetized with sodium thiamylal and sacrificed with a lethal dose of potassium chloride. The heart was removed and placed in $10 \%$ formalin for a 3-day period to facilitate sectioning. The atrial tissue, right ventricle, pericardial fat, aorta, and large surface vessels were then dissected from the left ventricle. The mean heart weight was $160 \pm 12 \mathrm{~g}$ and mean left ventricular weight was $105 \pm 29 \mathrm{~g}$. The left ventricle was sectioned into four transverse rings of approximately equal thickness (Fig. 1). The two central sections which constituted $61 \pm 7 \%$ of the left ventricular weight were then divided into six regions: anterior wall, intraventricular septum, posterior free wall, posterior papillary muscle region, lateral wall, and anterior papillary muscle region. Each region was then sectioned into four equal transmural layers from the epicardial to the endocardial surface (Fig. $1)$, weighed, and placed in vials for counting. For the remainder of this paper, these layers will be referred to as "layers 1 to 4," layer 1 being the most epicardial layer and layer 4 the most endocardial layer. Individual sample weights ranged from 0.72 to $3.94 \mathrm{~g}$ with most samples weighing $1.50-2.00 \mathrm{~g}$.

In five of the dogs studied a section of the base and apex of the right ventricular free wall was also sampled. Because of the smaller wall thickness, each section was cut into only two layers representing epicardial and endocardial myocardium.

The myocardial and blood reference samples were counted in a gamma spectrometer at window settings selected to

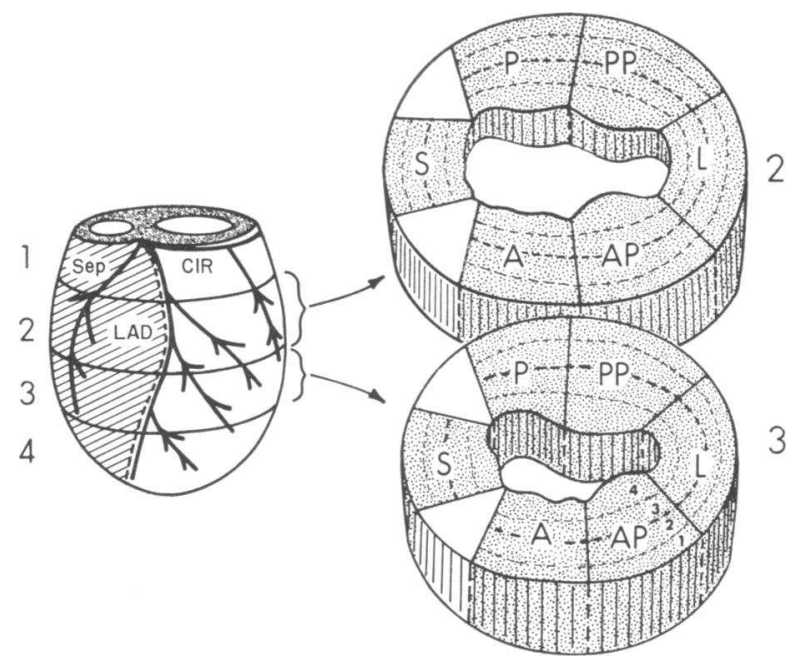

Figure 1 Illustrates the technique for sectioning the left ventricle. The atrial tissue and right ventricle were removed, as indicated by the stippled and lined area in the left hand figure. The left ventricle was then sectioned into four transverse sections and the two central sections, 2 and 3 , were subdivided into anterior free wall $(A)$, septum $(S)$, posterior free wall $(P)$, posterior papillary muscle region $(P P)$, lateral wall $(L)$, and anterior papillary muscle region $(A P)$. Each region was further subdivided into four equal layers from epicardium (layer 1) to endocardium (layer 4). 
correspond to the peak energies of each nuclide. The counts per minute recorded in each window were corrected for background and overlapping counts of the accompanying isotopes by a computer. ${ }^{8}$ Blood flow $(\mathrm{ml} / \mathrm{min})$ to each sample was computed by using the formula:

$$
F_{m}=F_{r} \cdot C_{m} / C_{r}
$$

where $F_{m}=$ sample blood flow $(\mathrm{ml} / \mathrm{min}), F_{r}=$ reference blood flow $(\mathrm{ml} / \mathrm{min}), C_{m}=$ counts per minute of each sample, $C_{r}=$ counts per minute of reference flow sample. Each sample blood flow ( $\mathrm{ml} / \mathrm{min}$ ) was divided by the sample weight and expressed as milliliter per minute per gram of myocardium.

The regional myocardial flows to rings 2 and 3 were compared by using multiple paired $t$ tests comparing each region and layer in ring 2 with the corresponding specimen in ring 3. The $P$ values were adjusted by the Bonferonni inequality (10) which corrects for performing multiple tests on correlated data, i.e., each $P$ value was multiplied by the number of paired $t$ tests performed on each set of data and a $P$ value of $\leq 0.05$ was required for statistical significance. Since no statistically significant difference in myocardial blood flow was found between any region in ring 2 and the corresponding region in ring 3 , data from corresponding regions of rings 2 and 3 were pooled for subsequent analysis. The transmural distribution of flow from epicardium to endocardium was compared by performing paired $t$ tests between layers 1 and 4 . Similarly, right ventricular flow distribution was analyzed by comparing the flow to endocardial and epicardial layers by using paired $t$ tests. These $P$ values were also corrected using the Bonferonni inequality.

Mean left ventricular flow for each intervention was determined by averaging the flow to all regions sampled in each dog, and regression equations for heart rate and the product of heart rate and mean arterial pressure plotted against mean left ventricular myocardial blood flow were computed. Mean right ventricular flow for each intervention was determined in the same manner for five dogs and the regression equation for heart rate plotted against mean right ventricular myocardial blood flow was computed. An analysis of covariance was performed to compare the right and left ventricular slopes.

\section{RESULTS}

Table I displays the mean hemodynamic data from nine dogs during resting conditions and three levels of treadmill exercise. During quiet resting conditions, heart rates ranged from 63 to 90 beats $/ \mathrm{min}$ and mean arterial pressures were $60-108 \mathrm{~mm} \mathrm{Hg}$. Left atrial pressures were 0-5 mm $\mathrm{Hg}$. During light exercise (heart rates 150-162 beats/min), mean arterial pressures ranged from 90 to $122 \mathrm{~mm} \mathrm{Hg}$ and were not significantly increased from rest $(P>0.1)$. During moderate exercise (heart rates $180-210$ beats/min), mean arterial pressures were $92-148 \mathrm{~mm} \mathrm{Hg}$. During heavy exercise (heart rates 222-270 beats/min), mean arterial pressures ranged from 100 to $138 \mathrm{~mm} \mathrm{Hg}$. Arterial pressures during both moderate and heavy exercise were significantly higher than at rest $(P<0.05)$. Left atrial pressures

\footnotetext{
${ }^{8}$ Model 1130, IBM Corp., Armonk, N. Y.
}

TABLE 1

Hemodynamic Data

\begin{tabular}{lccc}
\hline & Heart rate & $\begin{array}{c}\text { Mean aortic } \\
\text { pressure }\end{array}$ & $\begin{array}{c}\text { Mean left } \\
\text { atrial } \\
\text { pressure }\end{array}$ \\
\hline Rest & beats $/ \mathrm{min}$ & $\mathrm{mm} \mathrm{Hg}$ & $\mathrm{mm} \mathrm{Hg}$ \\
Light exercise & $76 \pm 3$ & $86 \pm 6$ & $2 \pm 1$ \\
Moderate exercise & $152 \pm 3 \ddagger$ & $102 \pm 4$ & $6 \pm 1$ \\
Heavy exercise & $190 \pm 3 \ddagger$ & $116 \pm 7^{*}$ & $4 \pm 1$ \\
& $240 \pm 6 \ddagger$ & $124 \pm 5 \ddagger$ & $5 \pm 2$ \\
\hline
\end{tabular}

Heart rate, mean aortic pressure, and mean left atrial pressure of all dogs studied at rest and during light, moderate, and heavy exercise. Values are mean \pm SEM. ${ }^{*}$ denotes $P<0.05$ and $\ddagger$ denotes $P<0.01$ in comparison with values at rest.

ranged from 1 to $12 \mathrm{~mm} \mathrm{Hg}$ and did not increase significantly during exercise.

Mean blood flow to all six regions and all transmural layers of the left ventricle showed linear increases with each level of exercise $(P<0.01)$. The plots of heart rate and the heart rate-mean arterial pressure product with mean left ventricular myocardial flow are shown in Figs. 2 and 3. The positive correlation between heart rate and left ventricular flow $(r=0.87)$ was not improved by comparing the heart rate-blood pressure product and left ventricular flow $(r=0.86)$.

Mean left ventricular regional myocardial blood flows during quiet resting conditions and three levels of treadmill exercise are plotted in Fig. 4. During rest, mean flow was $0.94 \pm 0.09 \mathrm{ml} / \mathrm{min} / \mathrm{g}$ and subendocardial flow exceeded subepicardial flow in all six regions. Endocardial/epicardial (endo/epi) flow ratios obtained by using layer 4/layer 1 flows were 1.12-1.33 (Table II) and significantly greater than $1.00(P<0.05)$ except in the anterior free wall and posterior papillary muscle regions.

Exercise resulted in increases in mean left ventricular flow from $1.83 \pm 0.11$ to $2.75 \pm 0.22$ to $3.90 \pm 0.26 \mathrm{ml} /$ $\mathrm{min} / \mathrm{g}$. Fig. 4 demonstrates that exercise resulted in increases in myocardial blood flow to each layer of every region of the left ventricle. However, light exercise produced greater increases in blood flow in layer 1 (mean increase $=0.94 \pm 0.08 \mathrm{ml} / \mathrm{min} / \mathrm{g}$ ) than in layer 4 (mean increase $=0.85 \pm 0.09 \mathrm{ml} / \mathrm{min} / \mathrm{g} ; P<0.02$ ), resulting in a significant decrease in mean endo/epi flow ratio from 1.23 at rest to 1.08 during light exercise $(P<0.05)$ (Table II). No additional change in endo/epi flow ratio occurred during moderate exercise (mean endo/epi = 1.08), while during heavy exercise a statistically insignificant additional decrease in mean endo/epi flow ratio occurred (mean endo/epi $=0.98 ; P>0.1$ ). During all three levels of exercise there was no significant difference between flow to the subepicardium and flow to the subendocardium of any region studied. 


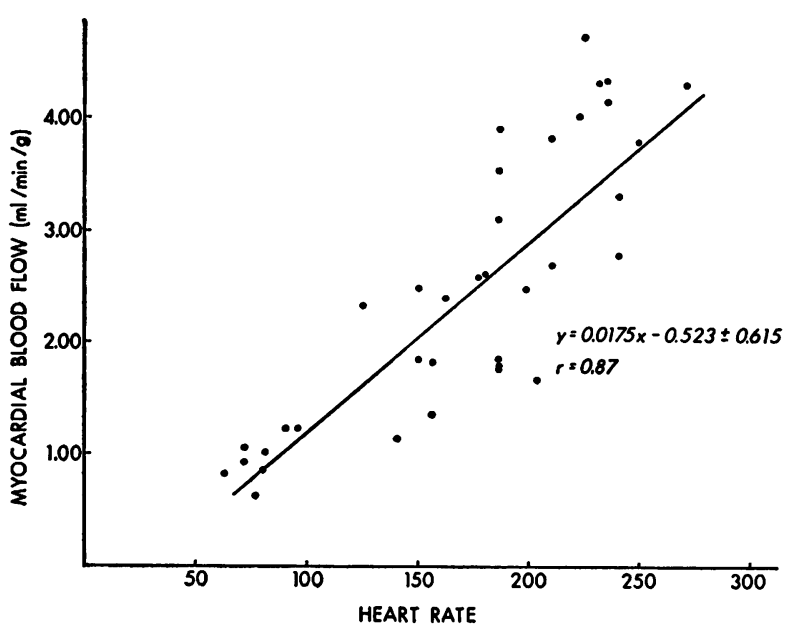

Figure 2 Mean left ventricular myocardial blood flow $(\mathrm{ml} / \mathrm{min} / \mathrm{g})$ plotted against heart rate (beats/min) for each dog studied at rest, and during light, moderate, and heavy exercise. The regression equation $\pm \mathrm{SE}$ of the estimate is shown.

Mean right ventricular myocardial blood flows during rest and three levels of exercise are presented in Table III. Right ventricular myocardial blood flow (RVF) at rest $(0.54 \pm 0.02 \mathrm{ml} / \mathrm{min} / \mathrm{g})$ also increased linearly with heart rate (HR) during each level of exercise from $1.20 \pm 0.07$ to $1.99 \pm 0.13$ to $3.11 \pm 0.19 \mathrm{ml} / \mathrm{min} / \mathrm{g}(\mathrm{RVF}=$ $0.015 \mathrm{HR}-0.846 \pm 0.495, r=0.90$ ). Analysis of covariance demonstrated that the slope of the right ventricular regression equation was not significantly different from the left ventricular regression equation $(P>0.4)$. Flow to regions near the apex showed the same increases as did regions near the base of the right ventricle. At rest and during each exercise level, mean right ventricular flow per gram of myocardium was significantly less than the corresponding left ventricular flow (right ventricular flow $=57-80 \%$ of left ventricular flow). Right ventricular endocardial flow was not different from epicardial flow during rest and all three levels of exercise $(P>$ 0.1 ).

\section{DISCUSSION}

The increases in mean myocardial blood flow during exercise in the present study were similar to those recorded in previous studies both in dogs in which proximal coronary artery blood flow was measured with electromagnetic flowmeters (1-4) and in humans where myocardial blood flow was estimated by the nitrous oxide saturation method (11). It is of interest that the regression equation relating mean left ventricular myocardial blood flow (MBF) to heart rate (HR) observed in the present study $(\mathrm{MBF}=0.0175 \mathrm{HR}-0.532, r=0.87)$ compares very well with the relationship observed in humans during upright exercise $(\mathrm{MBF}=0.0187 \mathrm{HR}$
$-0.650, r=0.82)(11)$. Thus, the absolute values for myocardial blood flow and the response of myocardial blood flow to exercise is remarkably similar in the human and in the dog.

Although coronary blood flow measured with electromagnetic or ultrasonic coronary artery flowmeters has been reported to increase in response to exercise, regional myocardial blood flow during exercise has not been measured previously. The radionuclide-labeled microsphere technique, utilized in the present study for estimation of regional myocardial blood flow, has been verified previously for measurement of both total organ flow and flow to small regions of left ventricular myocardium. In anesthetized open-chest dogs, using microspheres $15 \pm 5$ $\mu \mathrm{m}$ in diameter, Becker, Fortuin, and Pitt (12) observed left ventricular endo/epi blood flow ratios of 1.04 to 1.17. Similarly, Buckberg, Fixler, Archie, and Hoffman (13), using 7-10- $\mu \mathrm{m}$ microspheres in open-chest dogs, found endo/epi ratios of $1.01 \pm 0.07$. This uniform transmural distribution of small microspheres is similar to that observed with diffusible indicators such as ${ }^{80} \mathrm{RbCl}$ or antipyrine and appears to acceptably reflect distribution of myocardial blood flow (14-16).

Transmural myocardial blood flow distribution in resting awake dogs appears slightly different from that observed in open-chest animals in that subendocardial flow exceeds flow to the subepicardial myocardium in awake dogs. Thus, in the awake dogs in the present study, as well as those reported by Cobb, Bache, and Greenfield (17), the ratio of endo/epi blood flow was significantly greater than unity during resting conditions. That this does in fact represent a significant dif-

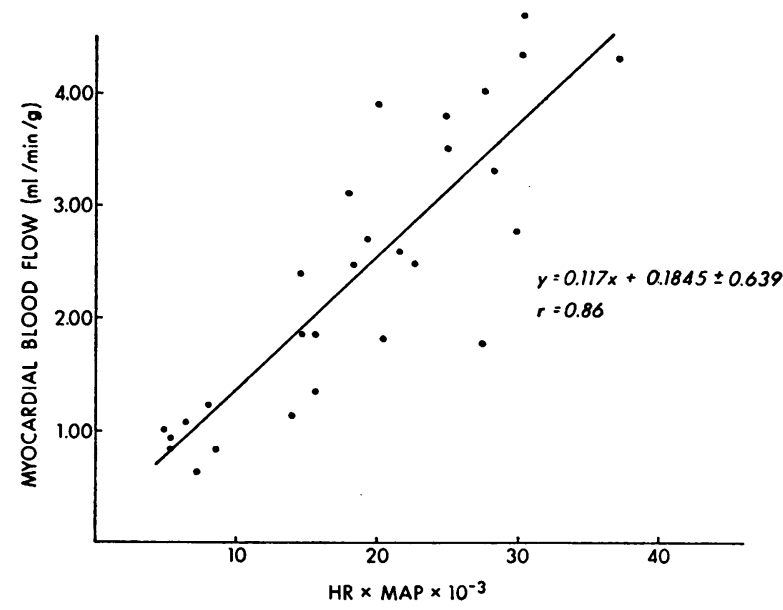

Figure 3 Mean left ventricular myocardial blood flow $(\mathrm{ml} / \mathrm{min} / \mathrm{g})$ plotted against the product of heart rate $X$ mean arterial pressure $\times 10^{-3}$ (beats $/ \mathrm{min} \times \mathrm{mm} \mathrm{Hg}$ ) for each dog studied at rest, and during light, moderate, and heavy exercise. The regression equation $\pm \mathrm{SE}$ of the estimate is shown. 

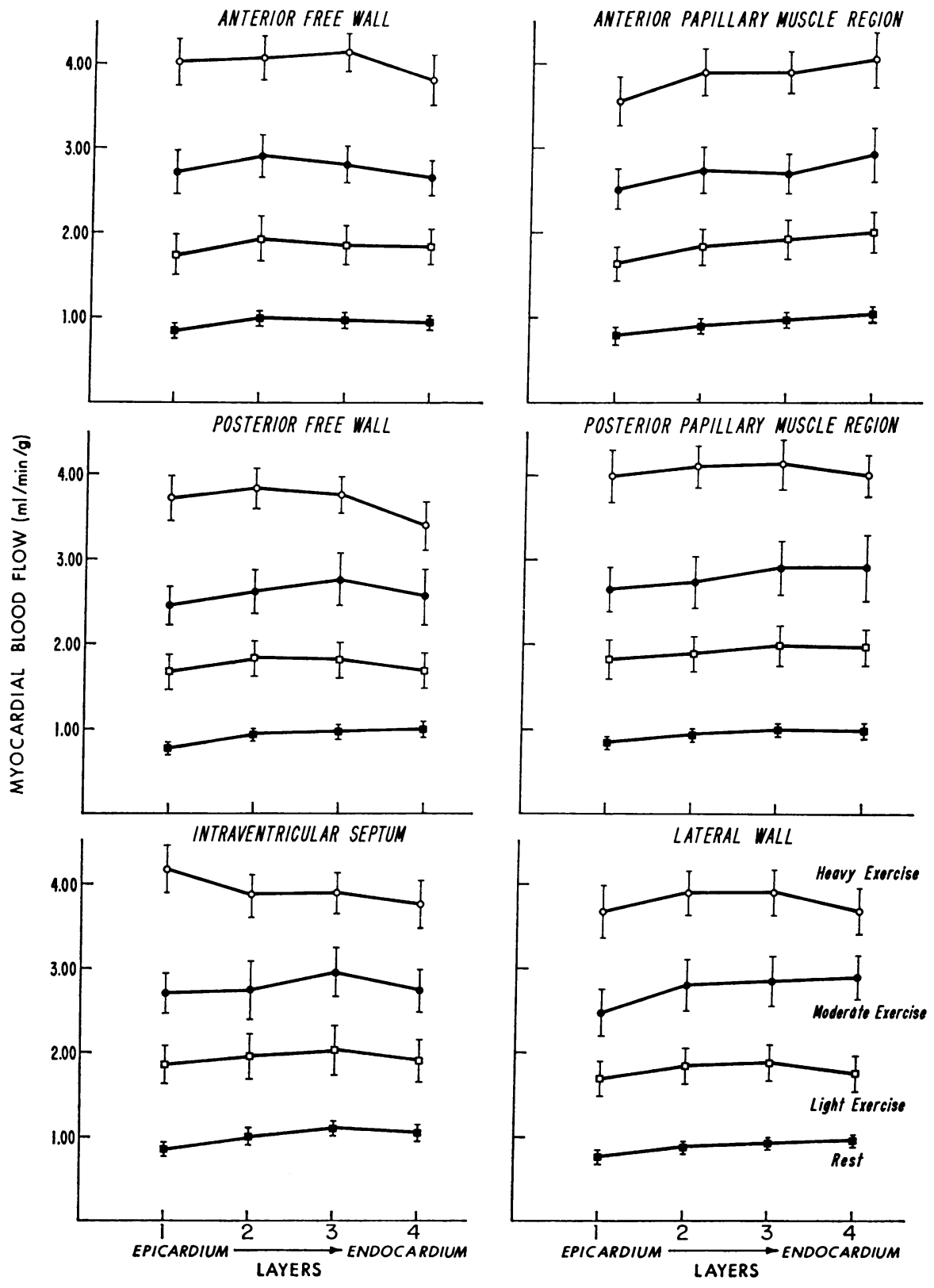

FIGURE 4 The mean left ventricular myocardial blood flow $(\mathrm{ml} / \mathrm{min} / \mathrm{g}) \pm \mathrm{SEM}$ to all regions and layers measured during rest, light exercise, moderate exercise, and heavy exercise.

ference in flow distribution between awake and openchest dogs was shown by Cobb et al. (17) who found that pentobarbital anesthesia and acute thoracotomy abolished the transmural gradient favoring the subendocardium. In the dogs reported in this study, all three levels of exercise also abolished the gradient of flow favoring the subendocardium which was present at rest. The reason that subendocardial blood flow exceeds subepicardial flow in the resting awake dog remains unclear. Likewise, the mechanisms resulting in abolition of this gradient during anesthesia or exercise are unknown and not necessarily similar for the two situations.

It is apparent from the data presented in Fig. 4 that subendocardial flow was able to increase regularly with each increasing level of exercise so that during light and moderate exercise the subendocardial vasculature clearly did possess the capacity for further vasodilation. Thus, at least during light and moderate exercise sub- 
TABLE II

Left Ventricular Endocardial/Epicardial Flow Ratios

\begin{tabular}{lcccccc}
\hline & Anterior & $\begin{array}{c}\text { Anterior } \\
\text { papillary } \\
\text { region }\end{array}$ & Posterior & $\begin{array}{c}\text { Posterior } \\
\text { papillary } \\
\text { region }\end{array}$ & Septum & Lateral \\
\hline Rest & 1.12 & $1.33 \ddagger$ & $1.27 \ddagger$ & 1.16 & $1.22^{*}$ & $1.26 \ddagger$ \\
Light exercise & 1.06 & $1.23^{*}$ & 1.00 & 1.09 & 1.03 & 1.05 \\
Moderate exercise & 0.97 & 1.16 & 1.04 & 1.10 & 1.01 & 1.18 \\
Heavy exercise & 0.95 & 1.14 & 0.91 & 1.00 & 0.90 & 1.00 \\
\hline
\end{tabular}

Tabulation of endocardial (layer 4)/epicardial (layer 1) flow ratios of each region of the left ventricle at rest and during light, moderate, and heavy exercise. $P$ values are for ratios significantly different from $1.00{ }^{*}$ denotes $P<0.05$ and $\ddagger$ denotes $P<0.01$ ).

endocardial flow potentially could have been maintained at a higher level to retain the gradient of flow favoring the subendocardium which was observed at rest. Since no attempt was made to determine whether subendocardial flow could be further increased during the heavy exercise period, it is possible that during this time subendocardial flow had approached its upper limit. Without some measurement of oxygen consumption or other metabolic parameter, one cannot be certain that equality of blood flow to the endocardium and epicardium represents "adequate" distribution of myocardial flow. However, the data do suggest that at least during the light and moderate exercise periods abolition of the gradient of flow favoring the subendocardium did not result from inability of the subendocardial vasculature to further augment flow.

Using intramyocardial polarographic electrodes, previous investigators $(7,8)$ have reported lower intramyocardial oxygen tensions in the subendocardium than in the subepicardium and have used this data to support the hypothesis that the subendocardium does not receive adequate blood flow to satisfy its metabolic needs. From these studies, they have suggested that the subendocardial vasculature is maximally dilated during resting conditions and thus unable to dilate further in re- sponse to increased oxygen demands. In contrast to this hypothesis, measurements of regional myocardial blood flow in the present study showed a marked ability of the coronary vasculature to increase blood flow in all transmural layers during exercise. Thus, the subendocardial vasculature was clearly not maximally dilated at rest, but was able to further dilate during exercise to maintain the gradient of diastolic flow favoring the subendocardium so that endocardial flow was not significantly different from epicardial flow.

In the present study, mean right ventricular flow per gram at rest was $57 \%$ of mean left ventricular flow. Since systolic tension development is a major determinant of myocardial oxygen consumption, the lower blood flow to the right ventricle may be related to the lower systolic tension of the right ventricle. Although the right ventricular myocardium received less blood at rest than the left ventricle, exercise resulted in similar increases in myocardial blood flow to both ventricles as evidenced by the similar slopes of the right and left ventricular regression plots of blood flow and heart rate. Furthermore, transmural blood flow to the right ventricular wall remained uniform during exercise.

Thus, exercise resulted in linear increases of myocardial blood flow to both right and left ventricles. Fur-

TABLE III

Myocardial Blood Flow to Right Ventricle

\begin{tabular}{|c|c|c|c|c|c|}
\hline & \multirow[b]{3}{*}{ Heart rate } & \multicolumn{4}{|c|}{ Right ventricular myocardial blood flow } \\
\hline & & \multicolumn{2}{|c|}{ Base } & \multicolumn{2}{|c|}{ Apex } \\
\hline & & Epi & Endo & Epi & Endo \\
\hline & beats $/ \min$ & \multicolumn{4}{|c|}{$\mathrm{ml} / \min / \mathrm{g}$} \\
\hline Rest & $78 \pm 4$ & $0.51 \pm 0.05$ & $0.57 \pm 0.05$ & $0.53 \pm 0.05$ & $0.55 \pm 0.03$ \\
\hline Light exercise & $156 \pm 3$ & $1.14 \pm 0.17$ & $1.22 \pm 0.20$ & $1.25 \pm 0.13$ & $1.18 \pm 0.16$ \\
\hline Moderate exercise & $185 \pm 2$ & $1.82 \pm 0.30$ & $1.99 \pm 0.33$ & $2.00 \pm 0.33$ & $2.16 \pm 0.30$ \\
\hline Heavy exercise & $242 \pm 11$ & $3.08 \pm 0.53$ & $3.08 \pm 0.50$ & $3.05 \pm 0.40$ & $3.23 \pm 0.55$ \\
\hline
\end{tabular}

$\mathrm{Mean} \pm \mathrm{SEM}$ of the heart rate and myocardial blood flow to the endocardial (endo) and epicardial (epi) layers of base and apex regions of the right ventricle at rest and during light, moderate, and heavy exercise. 
thermore, despite the tachycardia and coronary vasodilation associated with exercise, the coronary vasculature was able to increase blood flow to all regions of the right and left ventricles and still maintain a sufficient diastolic gradient of flow to sustain subendocardial blood flow equal to that in the subepicardium.

\section{ACKNOWLEDGMENTS}

The authors wish to acknowledge the valuable assistance of the following individuals in carrying out this study: Dr. Judith C. Rembert, Mr. Robert L. R. Wesly, Dr. Philip A. McHale, Mr. Kirby Cooper, Mr. Eric Fields, Mr. James Ferrell, Mr. Donald G. Powell, Ms. Linda Kohl, Mr. J. Michael Taylor and his staff of the Durham Veterans Administration Hospital Animal Care Facility, and Ms. Brenda Clement, Mrs. Brenda Haley, and Mrs. Rosa B. Ethridge for secretarial assistance.

This work was supported in part by National Institutes of Health grant HL-16438 from the U. S. Public Health Service, Veterans Administration Program No. 0517-01.

\section{REFERENCES}

1. Khouri, E. M., D. E. Gregg, and C. R. Rayford. 1965. Effect of exercise on cardiac output, left coronary flow and myocardial metabolism in the unanesthetized dog. Circ. Res. 17 : 427-437.

2. Vatner, S. F., C. B. Higgins, D. Franklin, and E. Braunwald. 1972. Role of tachycardia in mediating the coronary hemodynamic response to severe exercise. $J$. Appl. Physiol. 32: 380-385.

3. Van Citters, R. L., and D. Franklin. 1969. Cardiovascular performance of Alaska sled dogs during exercise. Circ. Res. 24: 33-42.

4. Vatner, S. F., C. B. Higgins, S. White, T. Patrick, and D. Franklin. 1971. The peripheral vascular response to severe exercise in untethered dogs before and after complete heart block. J. Clin. Invest. 50: 1950-1960.

5. Downey, J. M., and E. S. Kirk. 1974. Distribution of the coronary blood flow across the canine heart wall during systole. Circ. Res. 34: 251-257.

6. Moir, T. W. 1972. Subendocardial distribution of coro- nary blood flow and the effect of antianginal drugs. Circ. Res. 30: 621-627.

7. Kirk, E. S., and C. R. Honig. 1964. Nonuniform distribution of blood flow and gradients of oxygen tension within the heart. Am. J. Physiol. 207: 661-668.

8. Winbury, M. M. 1971. Redistribution of left ventricular blood flow produced by nitroglycerin. An example of integration of macro- and microcirculation. Circ. Res. Suppl. I. 28: 140-147.

9. Buckberg, G. D., J. C. Luck, D. B. Payne, J. I. E. Hoffman, J. P. Archie, and D. E. Fixler. 1971. Some sources of error in measuring regional blood flow with radioactive microspheres. J. Appl. Physiol. 31: 598-604.

10. Miller, R. G., Jr. 1966. Simultaneous Statistical Inference. McGraw-Hill Book Company, New York. 6769.

11. Kitamura, K., C. R. Jorgensen, F. L. Gobel, H. L. Taylor, and Y. Wang. 1972. Hemodynamic correlates of myocardial oxygen consumption during upright exercise. J. Appl. Physiol. 32: 516-522.

12. Becker, L. C., N. J. Fortuin, and B. Pitt. 1971. Effect of ischemia and antianginal drugs on the distribution of radioactive microspheres in the canine left venticle. Circ. Res. 28: 263-269.

13. Buckberg, G. D., D. E. Fixler, J. P. Archie, and J. I. E. Hoffman. 1972. Experimental subendocardial ischemia in dogs with normal coronary arteries. Circ. Res. $30: 67-81$.

14. Moir, T. W., and D. W. DeBra. 1965. Measurement of the endocardial distribution of left ventricular coronary blood flow by $\mathrm{Rb}^{86}$ chloride. Am. Heart J. 69: 795-800.

15. Utley, J., E. L. Carlson, J. I. E. Hoffman, H. M. Martinez, and G. D. Buckberg. 1974. Total and regional myocardial blood flow measurements with $25 \mu$, $15 \mu, 9 \mu$, and filtered $1-10 \mu$ diameter microspheres and antipyrine in dogs and sheep. Circ. Res. 34: 391-405.

16. Yipintsoi, T., W. A. Dobbs, Jr., P. D. Scanlon, T. J. Knopp, and J. B. Bassingthwaighte. 1973. Regional distribution of diffusible tracers and carbonized microspheres in the left ventricle of isolated dog hearts. Circ. Res. 33: 573-587.

17. Cobb, F. R., R. J. Bache, and J. C. Greenfield, Jr. 1974. Regional myocardial blood flow in awake dogs. J. Clin. Invest. 53: 1618-1625. 